\title{
An optimal dividends problem with transaction costs for spectrally negative Lévy processes
}

\author{
R.L. Loeffen * \\ Radon Institute for Computational and Applied Mathematics, \\ Austrian Academy of Sciences
}

December 12, 2008

\begin{abstract}
We consider an optimal dividends problem with transaction costs where the reserves are modeled by a spectrally negative Lévy process. We make the connection with the classical de Finetti problem and show in particular that when the Lévy measure has a log-convex density, then an optimal strategy is given by paying out a dividend in such a way that the reserves are reduced to a certain level $c_{1}$ whenever they are above another level $c_{2}$. Further we describe a method to numerically find the optimal values of $c_{1}$ and $c_{2}$.

AMS 2000 subject classifications. Primary 60J99; secondary 93E20, $60 \mathrm{G} 51$.

Keywords. Lévy process, stochastic control, impulse control, dividend problem, scale function
\end{abstract}

\section{Introduction}

In this paper we consider an offshoot of the classical de Finetti's optimal dividends problem in continuous time for which a transaction cost is incurred each time a dividend payment is made. Because of this fixed cost, it is no longer feasible to pay out dividends at a certain rate and therefore only lump sum dividend payments are possible.

Within this problem we assume that the underlying dynamics of the risk process is described by a spectrally negative Lévy process which is now widely accepted and used as a replacement for the classical Cramér-Lundberg process (cf. $[1,4,8-10,13,16,18,20,21,24])$. Recall that a Cramér-Lundberg risk process $\left\{X_{t}: t \geq 0\right\}$ corresponds to

$$
X_{t}=x+c t-\sum_{i=1}^{N_{t}} C_{i},
$$

\footnotetext{
*Postal address: Altenbergerstrasse $69, \quad$ A-4040 Linz, Austria. email: ronnie.loeffen@oeaw.ac.at
} 
where $x>0$ denotes the initial surplus, the claims $C_{1}, C_{2}, \ldots$ are i.i.d. positive random variables with expected value $\mu, c>0$ represents the premium rate and $N=\left\{N_{t}: t \geq 0\right\}$ is an independent Poisson process with arrival rate $\lambda$. Traditionally it is assumed in the Cramér-Lundberg model that the net profit condition $c>\lambda \mu$ holds, or equivalently that $X$ drifts to infinity. In this paper $X$ will be a general spectrally negative Lévy process and the condition that $X$ drifts to infinity will not be assumed.

We will now state the control problem considered in this paper. As mentioned before, $X=\left\{X_{t}: t \geq 0\right\}$ is a spectrally negative Lévy process which is defined on a filtered probability space $\left(\Omega, \mathcal{F}, \mathbb{F}=\left\{\mathcal{F}_{t}: t \geq 0\right\}, \mathbb{P}\right)$ satisfying the usual conditions. Within the definition of a spectrally negative Lévy process it is implicitly assumed that $X$ does not have monotone paths. We denote by $\left\{\mathbb{P}_{x}, x \in \mathbb{R}\right\}$ the family of probability measures corresponding to a translation of $X$ such that $X_{0}=x$, where we write $\mathbb{P}=\mathbb{P}_{0}$. Further $\mathbb{E}_{x}$ denotes the expectation with respect to $\mathbb{P}_{x}$ with $\mathbb{E}$ being used in the obvious way. The Lévy triplet of $X$ is given by $(\gamma, \sigma, \nu)$, where $\gamma \in \mathbb{R}, \sigma \geq 0$ and $\nu$ is a measure on $(0, \infty)$ satisfying

$$
\int_{(0, \infty)}\left(1 \wedge x^{2}\right) \nu(\mathrm{d} x)<\infty
$$

Note that even though $X$ only has negative jumps, for convenience we choose the Lévy measure to have only mass on the positive instead of the negative half line. The Laplace exponent of $X$ is given by

$$
\psi(\theta)=\log \left(\mathbb{E}\left(\mathrm{e}^{\theta X_{1}}\right)\right)=\gamma \theta+\frac{1}{2} \sigma^{2} \theta^{2}-\int_{(0, \infty)}\left(1-\mathrm{e}^{-\theta x}-\theta x \mathbf{1}_{\{0<x<1\}}\right) \nu(\mathrm{d} x)
$$

and is well defined for $\theta \geq 0$. Note that the Cramér-Lundberg process corresponds to the case that $\sigma=0, \nu(\mathrm{d} x)=\lambda F(\mathrm{~d} x)$ where $F$ is the law of $C_{1}$ and $\gamma=c-\int_{(0,1)} x \nu(\mathrm{d} x)$. The process $X$ will represent the risk process/reserves of the company before dividends are deducted.

We denote a dividend or control strategy by $\pi$, where $\pi=\left\{L_{t}^{\pi}: t \geq 0\right\}$ is a non-decreasing, left-continuous $\mathbb{F}$-adapted process which starts at zero. Further we assume that the process $L^{\pi}$ is a pure jump process, i.e.

$$
L_{t}^{\pi}=\sum_{0 \leq s<t} \Delta L_{s}^{\pi} \quad \text { for all } t \geq 0
$$

Here we mean by $\Delta L_{s}^{\pi}=L_{s+}^{\pi}-L_{s}^{\pi}$ the jump of the process $L^{\pi}$ at time $s$.

The random variable $L_{t}^{\pi}$ will represent the cumulative dividends the company has paid out until time $t$ under the control $\pi$. We define the controlled (net) risk process $U^{\pi}=\left\{U_{t}^{\pi}: t \geq 0\right\}$ by $U_{t}^{\pi}=X_{t}-L_{t}^{\pi}$. Let $\sigma^{\pi}=\inf \left\{t>0: U_{t}^{\pi}<0\right\}$ be the ruin time and define the value function of a dividend strategy $\pi$ by

$$
v_{\pi}(x)=\mathbb{E}_{x}\left[\int_{0}^{\sigma^{\pi}} \mathrm{e}^{-q t} \mathrm{~d}\left(L_{t}^{\pi}-\sum_{0 \leq s<t} \beta \mathbf{1}_{\left\{\Delta L_{s}^{\pi}>0\right\}}\right)\right],
$$


where $q>0$ is the discount rate and $\beta>0$ is the transaction cost incurred for each dividend payment. Note that because of (2) we can write $v_{\pi}(x)=$ $\mathbb{E}_{x}\left[\sum_{0 \leq t<\sigma^{\pi}} \mathrm{e}^{-q t}\left(\Delta L_{t}^{\pi}-\beta \mathbf{1}_{\left\{\Delta L_{t}^{\pi}>0\right\}}\right)\right]$. By definition $v_{\pi}(x)=0$ for $x<0$. A strategy $\pi$ is called admissible if ruin does not occur due to a lump sum dividend payment, i.e. $\Delta L_{t}^{\pi} \leq U_{t}^{\pi}$ for $t<\sigma^{\pi}$. Let $\Pi$ be the set of all admissible dividend policies. The control problem consists of finding the optimal value function $v_{*}$ given by

$$
v_{*}(x)=\sup _{\pi \in \Pi} v_{\pi}(x)
$$

and an optimal strategy $\pi_{*} \in \Pi$ such that

$$
v_{\pi_{*}}(x)=v_{*}(x) \text { for all } x \geq 0 .
$$

Since control strategies of the form (2) are known as impulse controls, we refer to this problem as the impulse control problem.

An important type of strategy for the impulse control problem is the one we call in this paper the $\left(c_{1} ; c_{2}\right)$ policy and which is similar to the well known $(s, S)$ policy appearing in inventory control models, see e.g. [3,25]. The $\left(c_{1} ; c_{2}\right)$ policy is the strategy where each time the reserves are above a certain level $c_{2}$, a dividend payment is made which brings the reserves down to another level $c_{1}$ and where no dividends are paid out when the reserves are below $c_{2}$. In case $X$ is a Brownian motion plus drift, Jeanblanc and Shiryaev [14] showed that an optimal strategy for the impulse control problem is formed by a $\left(c_{1} ; c_{2}\right)$ policy. Paulsen [22] considered the case when $X$ is modeled by a diffusion process and showed that under certain conditions a $\left(c_{1} ; c_{2}\right)$ policy is optimal. Note that in Paulsen [22] this type of strategy is referred to as a lump sum dividend barrier strategy. Further, Alvarez and Rakkolainen [2] study the case where the driving process is a spectrally negative Lévy diffusion with a jump component of geometric form. In this paper we will investigate when an optimal strategy for our impulse control problem is formed by a $\left(c_{1} ; c_{2}\right)$ policy.

When the assumption (2) is dropped and the transaction cost $\beta$ is taken to be equal to zero, then the impulse control problem transforms into the classical de Finetti optimal dividends problem. The latter optimal dividends problem will be referred to as the de Finetti problem in the remainder of the paper. This particular problem was introduced by de Finetti [7] in a discrete time setting for the case that the risk process evolves as a simple random walk. Thereafter the de Finetti problem has been studied in a continuous time setting for the case that $X$ is a Cramér-Lundberg risk process $[5,11]$ and for the case that the risk process is a general spectrally negative Lévy process $[4,18,20]$. For this problem an important strategy is the so called barrier strategy. The barrier strategy at level $a$ is the strategy where initially (in case the starting value of the reserves are above $a$ ) a lump sum dividend payment is made to bring the reserves back to level $a$ and thereafter each time the reserves reach the level $a$, non-lump sum dividend payments are made in such a way that the reserves do not exceed the level $a$, but where no dividends are paid out when the reserves are strictly below 
a. Mathematically this corresponds to reflecting the risk process $X$ at $a$. The barrier strategy at level $a$ may be seen (at least intuitively) as a limit of $\left(c_{1} ; c_{2}\right)$ policies where $c_{1}$ and $c_{2}$ converge to the barrier $a$.

Gerber [11] proved that an optimal strategy for the de Finetti problem is formed by a barrier strategy in the case where $X$ is a Cramér-Lundberg risk process with exponentially distributed claims. Building on the work of Avram et al. [4], Loeffen [20] showed that optimality of the barrier strategy for the de Finetti problem depends on the shape of the so-called scale function of a spectrally negative Lévy process. To be more specific, the $q$-scale function of $X$, $W^{(q)}: \mathbb{R} \rightarrow[0, \infty)$ where $q \geq 0$, is the unique function such that $W^{(q)}(x)=0$ for $x<0$ and on $[0, \infty)$ is a strictly increasing and continuous function characterized by its Laplace transform which is given by

$$
\int_{0}^{\infty} \mathrm{e}^{-\theta x} W^{(q)}(x) \mathrm{d} x=\frac{1}{\psi(\theta)-q} \quad \text { for } \theta>\Phi(q),
$$

where $\Phi(q)=\sup \{\theta \geq 0: \psi(\theta)=q\}$ is the right-inverse of $\psi$. Theorem 2 of Loeffen [20] then says that if $W^{(q)}$ is sufficiently smooth and if $W^{(q) \prime}$ is increasing on $\left(a^{*}, \infty\right)$ where $a^{*}$ is the largest point where $W^{(q) \prime}$ attains its global minimum, then the barrier strategy at $a^{*}$ is optimal for the de Finetti problem. Here $W^{(q)}$ being sufficiently smooth means that $W^{(q)}$ is once/twice continuously differentiable when $X$ is of bounded/unbounded variation. It was then shown in [20] that when $X$ has a Lévy measure which has a completely monotone density, these conditions on the scale function are satisfied and in particular that $W^{(q) '}$ is strictly convex on $(0, \infty)$. (Note that it was shown in $[21]$ that $W^{(q) \prime}$ is actually strictly log-convex.) Shortly thereafter, Kyprianou et al. [18] proved that $W^{(q) \prime}$ is strictly convex on $\left(a^{*}, \infty\right)$ under the weaker condition that the Lévy measure has a density which is log-convex and then used Theorem 2 from [20] mentioned above, to conclude that the barrier strategy at $a^{*}$ is optimal (though they needed to relax the sufficiently smoothness assumption). It is important to note that without a condition on the Lévy measure the barrier strategy is not optimal in general. Indeed Azcue and Muler [5] have given an example for which no barrier strategy is optimal.

In this paper we will show that the results for the de Finetti problem mentioned in the previous paragraph have their counterparts for the impulse control problem, whereby the role of the barrier strategy is now played by the $\left(c_{1} ; c_{2}\right)$ policy. In particular we will give a theorem similar to Theorem 2 in [20] and then use this theorem to show that a certain $\left(c_{1} ; c_{2}\right)$ policy is optimal if the Lévy measure has a log-convex density. Moreover we give an example for which no $\left(c_{1} ; c_{2}\right)$ policy is optimal.

The outline of this paper is as follows. In the next section we review some properties concerning scale functions and in Section 3 we give sufficient conditions under which the $\left(c_{1} ; c_{2}\right)$ policy is optimal. We treat the case when the Lévy measure has a log-convex density in Section 4 and show that the optimal strategy is formed by a unique $\left(c_{1} ; c_{2}\right)$ policy. Further we show how to 
numerically find the optimal values of $c_{1}$ and $c_{2}$. In the last section we treat two explicit examples including one for which we show that no $\left(c_{1} ; c_{2}\right)$ policy is optimal.

\section{Scale functions}

The scale function, defined via its Laplace transform given by (3), appears in almost all fluctuation identities for spectrally negative Lévy processes. As an example we mention the two sided exit above problem for which

$$
\mathbb{E}_{x}\left(\mathrm{e}^{-q \tau_{a}^{+}} \mathbf{1}_{\left(\tau_{a}^{+}<\tau_{0}^{-}\right)}\right)=\frac{W^{(q)}(x)}{W^{(q)}(a)},
$$

where $x \leq a, \tau_{0}^{-}=\inf \left\{t>0: X_{t}<0\right\}$ and $\tau_{a}^{+}=\inf \left\{t>0: X_{t}>a\right\}$. For background on scale functions we refer to Chapter 8 of Kyprianou [15].

We will now recall some properties of scale functions which we will need later on. The initial value of the scale function $W^{(q)}(0)$ is equal to $1 / c$ when $X$ is of bounded variation and is equal to 0 when $X$ is of unbounded variation. Here $c=\gamma+\int_{0}^{1} x \nu(\mathrm{d} x)$ stands for the drift of $X$ when it is of bounded variation. The initial value of the derivative of the scale function is given by (see e.g. [19])

$$
W^{(q) \prime}(0):=\lim _{x \downarrow 0} W^{(q) \prime}(x)= \begin{cases}2 / \sigma^{2} & \text { when } \sigma>0 \\ (\nu(0, \infty)+q) / c^{2} & \text { when } \sigma=0 \text { and } \nu(0, \infty)<\infty \\ \infty & \text { otherwise. }\end{cases}
$$

The scale function is log-concave for all $q \geq 0$ (see e.g. [21]) and thus $\frac{W^{(q) \prime}(x)}{W^{(q)}(x)}$ is a decreasing function (in the weak sense). For $q \geq 0$ there is the following relation between scale functions

$$
W^{(q)}(x)=\mathrm{e}^{\Phi(q) x} W_{\Phi(q)}(x),
$$

where $W_{\Phi(q)}$ is the (0-)scale function of $X$ under the measure $\mathbb{P}^{\Phi(q)}$ defined by

$$
\left.\frac{\mathrm{d} \mathbb{P}^{\Phi(q)}}{\mathrm{d} \mathbb{P}}\right|_{\mathcal{F}_{t}}=\mathrm{e}^{\Phi(q) X_{t}-q t} .
$$

When the Lévy measure has a density which is log-convex, Kyprianou et al. [18] proved that $W^{(q) \prime}$ is strictly increasing and strictly convex on $\left(a^{*}, \infty\right)$, where $a^{*}$ is defined (as in Section 1) by

$$
a^{*}=\sup \left\{a \geq 0: W^{(q) \prime}(a) \leq W^{(q) \prime}(x) \text { for all } x \geq 0\right\}
$$

which is necessarily finite since $\lim _{x \rightarrow \infty} W^{(q)^{\prime}}(x)=\infty$. Note also that by the log-concavity of $W^{(q)}$, it follows that $W^{(q) \prime}\left(a^{*}\right)>0$. In the next proposition we show that slightly more can be said in this case. 
Proposition 1. If the Lévy measure has a log-convex density, then there exists $0 \leq a^{\prime} \leq a^{*}$ such that $W^{(q) \prime}$ is strictly decreasing on $\left(0, a^{\prime}\right)$, constant on $\left(a^{\prime}, a^{*}\right)$ and strictly increasing and strictly convex on $\left(a^{*}, \infty\right)$.

Proof. Under the condition that the Lévy measure has a log-convex density, Kyprianou, Rivero \& Song [18, Proof of Theorem 2.6] proved that $u_{q}(x)=$ $\mathrm{e}^{\Phi(q) x} W_{\Phi(q)}^{\prime}(x)$ is convex on $(0, \infty)$. Therefore the function $k:(0, \infty) \rightarrow \mathbb{R}$ defined by $k(y)=\Phi(q) W^{(q) \prime}(y)+u_{q}^{\prime+}(y)$ is well defined and $u_{q}^{\prime+}$ is an increasing function. Here $u_{q}^{\prime+}$ stands for the right-derivative of $u_{q}$. Using (5) we can write for arbitrary $a>0$

$$
\begin{aligned}
k(y)= & \mathrm{e}^{\Phi(q) y}\left\{\Phi^{2}(q) W_{\Phi(q)}(y)+2 \Phi(q) W_{\Phi(q)}^{\prime}(y)+W_{\Phi(q)}^{\prime \prime+}(y)\right\} \\
= & \mathrm{e}^{\Phi(q) y}\left\{u_{q}^{\prime+}(y) \mathrm{e}^{-\Phi(q) y}+\int_{a}^{y} u_{q}^{\prime+}(z) \Phi(q) \mathrm{e}^{-\Phi(q) z} \mathrm{~d} z\right. \\
& \left.+\Phi^{2}(q) W_{\Phi(q)}(a)+\Phi(q) W_{\Phi(q)}^{\prime}(a)\right\} .
\end{aligned}
$$

Suppose now that $x, y>0$ with $x \leq y$ and let

$$
M=\frac{\int_{x}^{y} u_{q}^{\prime+}(z) \Phi(q) \mathrm{e}^{-\Phi(q) z} \mathrm{~d} z}{\mathrm{e}^{-\Phi(q) x}-\mathrm{e}^{-\Phi(q) y}} .
$$

Since $u_{q}^{\prime+}$ is an increasing function, it follows that $M \in\left[u_{q}^{\prime+}(x), u_{q}^{\prime+}(y)\right]$ and hence we deduce

$$
\begin{aligned}
& k(y) \mathrm{e}^{-\Phi(q) y}-k(x) \mathrm{e}^{-\Phi(q) x} \\
& =u_{q}^{\prime+}(y) \mathrm{e}^{-\Phi(q) y}-u_{q}^{\prime+}(x) \mathrm{e}^{-\Phi(q) x}+M\left(\mathrm{e}^{-\Phi(q) x}-\mathrm{e}^{-\Phi(q) y}\right) \\
& \quad=\left(u_{q}^{\prime+}(y)-M\right) \mathrm{e}^{-\Phi(q) y}+\left(M-u_{q}^{\prime+}(x)\right) \mathrm{e}^{-\Phi(q) x} \geq 0 .
\end{aligned}
$$

Hence $y \mapsto k(y) \mathrm{e}^{-\Phi(q) y}$ is an increasing function and it follows that there exists $0 \leq a_{1} \leq a_{2} \leq \infty$ such that $k$ is strictly negative on $\left(0, a_{1}\right)$, zero on $\left(a_{1}, a_{2}\right)$ and strictly positive and strictly increasing on $\left(a_{2}, \infty\right)$. Since we can use (5) to write for arbitrary $a>0$

$$
W^{(q) \prime}(x)=\Phi(q) W^{(q)}(x)+u_{q}(x)=W^{(q) \prime}(a)+\int_{a}^{x} k(y) \mathrm{d} y \quad \text { for } x>0,
$$

the statement of the proposition follows with $a^{\prime}=a_{1}$ and $a^{*}=a_{2}$. Note also that the fact that $\lim _{x \rightarrow \infty} W^{(q) \prime}(x)=\infty$ forces $a_{2}$ to be finite.

Despite the fact that the scale function is in general only implicitly known through its Laplace transform, there are plenty examples of spectrally negative Lévy processes for which there exists closed-form expressions for their scale functions, although most of these examples only deal with the $q=0$ scale function. In case no explicit formula for the scale function exists, one can use numerical methods as described in [26] to invert the Laplace transform of the scale function. We refer to the papers $[12,17,18]$ for an updated account on explicit examples of scale functions and their properties. 


\section{Conditions for optimality of a $\left(c_{1} ; c_{2}\right)$ policy}

In this section the main result will be a theorem which gives sufficient conditions on the scale function $W^{(q)}$ such that a certain $\left(c_{1} ; c_{2}\right)$ policy is optimal for the impulse control problem; this result is similar to Theorem 2 in [20] which concerns optimality of the barrier strategy at $a^{*}$ for the de Finetti problem. The way we show this result is similar to the approach taken in [4], [20] and [21], namely we first calculate the value function of a parameterized class of strategies (in our case the class of $\left(c_{1} ; c_{2}\right)$ policies), then we choose the (candidate) optimal strategy from this class by optimizing over the parameters and finally we put this particular strategy through a verification lemma. The main additional complexity compared to the de Finetti problem is to characterize the optimal values of the parameters $c_{1}$ and $c_{2}$ which is a 2-dimensional optimization problem, whereas finding the optimal barrier strategy is just a 1-dimensional one.

We first derive the value function corresponding to a $\left(c_{1} ; c_{2}\right)$ policy. A description of a $\left(c_{1} ; c_{2}\right)$ policy was given in Section 1 . We now define this strategy mathematically. For $c_{2}>c_{1} \geq 0$, let $\left\{T_{i}^{c_{1}, c_{2}}, i=1,2, \ldots\right\}$ be the set of stopping times defined by

$$
T_{i}^{c_{1}, c_{2}}=\inf \left\{t>0: X_{t}>X_{0} \vee c_{2}+\left(c_{2}-c_{1}\right)(i-1)\right\}, \quad i=1,2, \ldots
$$

Then $\pi_{c_{1}, c_{2}}=\left\{L_{t}^{c_{1}, c_{2}}: t \geq 0\right\}$ is defined by

$$
L_{t}^{c_{1}, c_{2}}=\mathbf{1}_{\left\{T_{1}^{c_{1}, c_{2}}<t\right\}}\left(X_{0} \vee c_{2}-c_{1}\right)+\sum_{i=2}^{\infty} \mathbf{1}_{\left\{T_{i}^{c_{1}, c_{2}}<t\right\}}\left(c_{2}-c_{1}\right), \quad t \geq 0
$$

Note that with $U_{t}^{c_{2}, c_{2}}=X_{t}-L_{t}^{c_{1}, c_{2}}$ the above defined stopping times can then be identified as $T_{1}^{c_{1}, c_{2}}=\inf \left\{t>0: U_{t}^{c_{1}, c_{2}}>c_{2}\right\}$ and $T_{i+1}^{c_{1}, c_{2}}=\inf \left\{t>T_{i}^{c_{1}, c_{2}}\right.$ : $\left.U_{t}^{c_{1}, c_{2}}>c_{2}\right\}$ for $i \geq 1$. Let $v_{c_{1}, c_{2}}$ denote the value function of the strategy $\pi_{c_{1}, c_{2}}$.

Proposition 2. The value function of the strategy $\pi_{c_{1}, c_{2}}$ is given by

$$
v_{c_{1}, c_{2}}(x)= \begin{cases}\frac{c_{2}-c_{1}-\beta}{W^{(q)}\left(c_{2}\right)-W^{(q)}\left(c_{1}\right)} W^{(q)}(x) & \text { if } x \leq c_{2}, \\ x-c_{1}-\beta+\frac{c_{2}-c_{1}-\beta}{W^{(q)}\left(c_{2}\right)-W^{(q)}\left(c_{1}\right)} W^{(q)}\left(c_{1}\right) & \text { if } x>c_{2} .\end{cases}
$$

Proof. Since $U^{c_{1}, c_{2}}$ is a Markov process, the proposition only needs to be proved for $0 \leq x \leq c_{2}$. Let $x \in\left[0, c_{2}\right]$. Since no dividends are paid out until $X$ reaches the level $c_{2}$, we get by applying the strong Markov property at $\tau_{c_{2}}^{+}$and (4)

$$
v_{c_{1}, c_{2}}(x)=\mathbb{E}_{x}\left(\mathrm{e}^{-q \tau_{c_{2}}^{+}} \mathbf{1}_{\left\{\tau_{c_{2}}^{+}<\tau_{0}^{-}\right\}}\right) v_{c_{1}, c_{2}}\left(c_{2}\right)=\frac{W^{(q)}(x)}{W^{(q)}\left(c_{2}\right)} v_{c_{1}, c_{2}}\left(c_{2}\right) .
$$

When $X_{0}=c_{2}$, a dividend payment of size $c_{2}-c_{1}$ is made immediately plus a transaction cost of size $\beta$ is incurred and so by using the above equation we get

$$
v_{c_{1}, c_{2}}\left(c_{2}\right)=c_{2}-c_{1}-\beta+v_{c_{1}, c_{2}}\left(c_{1}\right)=c_{2}-c_{1}-\beta+\frac{W^{(q)}\left(c_{1}\right)}{W^{(q)}\left(c_{2}\right)} v_{c_{1}, c_{2}}\left(c_{2}\right) \text {. }
$$


Now solving for $v_{c_{1}, c_{2}}\left(c_{2}\right)$ and plugging the result in (6) will give us the desired expression for $v_{c_{1}, c_{2}}(x)$.

We now want to find the values of $\left(c_{1} ; c_{2}\right)$ which is likely to give us the best $\left(c_{1} ; c_{2}\right)$ policy. A good guess would be the values of $\left(c_{1} ; c_{2}\right)$ that minimizes

$$
g\left(c_{1}, c_{2}\right)=\frac{W^{(q)}\left(c_{2}\right)-W^{(q)}\left(c_{1}\right)}{c_{2}-c_{1}-\beta}
$$

where the domain of $g$ is given by $\operatorname{dom}(g)=\left\{\left(c_{1} ; c_{2}\right): c_{1} \geq 0, c_{2}>c_{1}+\beta\right\}$. Let $C^{*}$ be the set of minimizers of $g$, i.e.

$$
C^{*}=\left\{\left(c_{1}^{*} ; c_{2}^{*}\right) \in \operatorname{dom}(g): g\left(c_{1}^{*}, c_{2}^{*}\right)=\inf _{\left(c_{1} ; c_{2}\right) \in \operatorname{dom}(g)} g\left(c_{1}, c_{2}\right)\right\}
$$

Proposition 3. Assume $W^{(q)} \in C^{1}(0, \infty)$. Then the set $C^{*}$ is non-empty and for each $\left(c_{1}^{*} ; c_{2}^{*}\right) \in C^{*}$ we have

$$
W^{(q) \prime}\left(c_{2}^{*}\right)=\frac{W^{(q)}\left(c_{2}^{*}\right)-W^{(q)}\left(c_{1}^{*}\right)}{c_{2}^{*}-c_{1}^{*}-\beta}
$$

and further one of the following holds: (i) $W^{(q)^{\prime}}\left(c_{1}^{*}\right)=W^{(q) \prime}\left(c_{2}^{*}\right)$ or (ii) $c_{1}^{*}=0$.

Proof. First, by the mean value theorem

$$
g\left(c_{1}, c_{2}\right) \geq \min _{x \in\left[c_{1}, c_{2}\right]} W^{(q)^{\prime}}(x) \frac{c_{2}-c_{1}}{c_{2}-c_{1}-\beta}>\min _{x \in\left[c_{1}, c_{2}\right]} W^{(q) \prime}(x)
$$

and since $\lim _{x \rightarrow \infty} W^{(q)^{\prime}}(x)=\infty$, this implies that an infimum of $g$ is not reached when $c_{1} \rightarrow \infty$. Hence there exists $C_{1}>0$ such that

$$
\inf _{\operatorname{dom}(g)} g=\inf _{\operatorname{dom}(g), c_{1} \leq C_{1}} g\left(c_{1}, c_{2}\right) .
$$

Second,

$$
\begin{aligned}
\lim _{c_{2} \rightarrow \infty} \inf _{c_{1} \in\left[0, C_{1}\right]} g\left(c_{1}, c_{2}\right) & =\lim _{c_{2} \rightarrow \infty} \inf _{c_{1} \in\left[0, C_{1}\right]}\left(\frac{W^{(q)}\left(c_{2}\right)}{c_{2}-c_{1}-\beta}-\frac{W^{(q)}\left(c_{1}\right)}{c_{2}-c_{1}-\beta}\right) \\
& \geq \lim _{c_{2} \rightarrow \infty}\left(\frac{W^{(q)}\left(c_{2}\right)}{c_{2}-\beta}-\frac{W^{(q)}\left(C_{1}\right)}{c_{2}-C_{1}-\beta}\right)=\infty
\end{aligned}
$$

and hence an infimum of $g$ is also not reached when $c_{2} \rightarrow \infty$. Finally, by the mean value theorem

$$
g\left(c_{1}, c_{2}\right) \geq \frac{W^{(q)^{\prime}}\left(a^{*}\right)\left(c_{2}-c_{1}\right)}{c_{2}-c_{1}-\beta} \geq W^{(q)^{\prime}}\left(a^{*}\right) \frac{\beta}{c_{2}-c_{1}-\beta}
$$

and thus since $W^{(q)^{\prime}}\left(a^{*}\right)>0$, an infimum of $g$ is not reached when $\left(c_{1} ; c_{2}\right)$ converges to the line $c_{2}=c_{1}+\beta$. 
By the previous conclusions and the continuity of $g$ it follows that $C^{*}$ is non-empty and that for each $\left(c_{1}^{*} ; c_{2}^{*}\right) \in C^{*}$ either $c_{1}^{*}=0$ or $\left(c_{1}^{*} ; c_{2}^{*}\right)$ is an interior point of $\operatorname{dom}(g)$. In the latter case it follows since $g$ is partial differentiable in $c_{1}$ and $c_{2}$ (which follows from the hypothesis $W^{(q)} \in C^{1}(0, \infty)$ ) that

$$
\frac{\partial g\left(c_{1}, c_{2}\right)}{\partial c_{1}}\left(c_{1}^{*}, c_{2}^{*}\right)=0 \quad \text { and } \quad \frac{\partial g\left(c_{1}, c_{2}\right)}{\partial c_{2}}\left(c_{1}^{*}, c_{2}^{*}\right)=0
$$

which in turn implies (7) and (i). In the case that $c_{1}^{*}=0$, we have that $c_{2}^{*}$ minimizes the function $g_{0}:(\beta, \infty) \rightarrow(0, \infty)$ defined by $g_{0}\left(c_{2}\right)=g\left(0, c_{2}\right)=$ $\frac{W^{(q)}\left(c_{2}\right)-W^{(q)}(0)}{c_{2}-\beta}$ and hence $g_{0}^{\prime}\left(c_{2}^{*}\right)=0$ which implies $(7)$.

Corollary 4. Assume $W^{(q)} \in C^{1}(0, \infty)$. Then for each $\left(c_{1}^{*} ; c_{2}^{*}\right)$ in $C^{*}$

$$
v_{c_{1}^{*}, c_{2}^{*}}(x)= \begin{cases}\frac{W^{(q)}(x)}{W^{(q) \prime}\left(c_{2}^{*}\right)} & \text { for } x \leq c_{2}^{*}, \\ x-c_{2}^{*}+\frac{W^{(q)}\left(c_{2}^{*}\right)}{W^{(q) \prime}\left(c_{2}^{*}\right)} & \text { for } x>c_{2}^{*}\end{cases}
$$

and so $v_{c_{1}^{*}, c_{2}^{*}}(x)=v_{c_{2}^{*}}(x)$, where $v_{c_{2}^{*}}$ is the value of the barrier strategy at level $c_{2}^{*}$ in the de Finetti problem. Moreover, $W^{(q) \prime}\left(c_{2}^{*}\right)>W^{(q) \prime}\left(a^{*}\right)$.

Proof. The corollary follows directly from the two previous propositions and (8). Note that the formula for the value of a barrier strategy was given by Avram et al. [4].

Corollary (4) shows that the value function of any $\left(c_{1} ; c_{2}\right)$ policy with $\left(c_{1} ; c_{2}\right)$ belonging to $C^{*}$ is equal to the value function of a barrier strategy; in particular this means that the derivative of the value function at $c_{2}^{*}$ is equal to one (the marginal value of paying dividend) and thus $v_{c_{1}^{*}, c_{2}^{*}}$ is continuously differentiable. This will later allow us to use results for barrier strategies in order to solve the impulse control problem.

A necessary condition for a certain strategy $\pi$ to be optimal is that the extra gain obtained by starting with a higher initial capital $x$ compared to starting with a lower amount of initial capital $y$, should not be less than the gain generated by paying out immediately a dividend which brings you from level $x$ down to level $y$. In the next lemma we show that the strategy $\pi_{c_{1}^{*}, c_{2}^{*}}$ with $\left(c_{1}^{*} ; c_{2}^{*}\right) \in C^{*}$ satisfies this necessary condition.

Lemma 5. Assume $W^{(q)} \in C^{1}(0, \infty)$ and let $\left(c_{1}^{*} ; c_{2}^{*}\right) \in C^{*}$. Then for $x \geq y \geq 0$,

$$
v_{c_{1}^{*}, c_{2}^{*}}(x)-v_{c_{1}^{*}, c_{2}^{*}}(y) \geq x-y-\beta .
$$

Proof. Note that since $v_{c_{1}^{*}, c_{2}^{*}}$ is an increasing function, we can assume without loss of generality that $x-y>\beta$. First suppose $x \geq y \geq c_{2}^{*}$, then $v_{c_{1}^{*}, c_{2}^{*}}(x)-$ $v_{c_{1}^{*}, c_{2}^{*}}(y)=x-y \geq x-y-\beta$. Second, if $c_{2}^{*} \geq x \geq y$, then

$$
v_{c_{1}^{*}, c_{2}^{*}}(x)-v_{c_{1}^{*}, c_{2}^{*}}(y)=\frac{W^{(q)}(x)-W^{(q)}(y)}{W^{(q)^{\prime}}\left(c_{2}^{*}\right)} \geq x-y-\beta,
$$


where the inequality follows since $\left(c_{1}^{*} ; c_{2}^{*}\right) \in C^{*}$ and therefore with the help of (7)

$$
W^{(q) \prime}\left(c_{2}^{*}\right)=\frac{W^{(q)}\left(c_{2}^{*}\right)-W^{(q)}\left(c_{1}^{*}\right)}{c_{2}^{*}-c_{1}^{*}-\beta} \leq \frac{W^{(q)}(x)-W^{(q)}(y)}{x-y-\beta} .
$$

Finally, suppose $x \geq c_{2}^{*} \geq y$, then using Corollary 4

$$
\begin{aligned}
v_{c_{1}^{*}, c_{2}^{*}}(x)-v_{c_{1}^{*}, c_{2}^{*}}(y) & =x-c_{2}^{*}+\frac{W^{(q)}\left(c_{2}^{*}\right)-W^{(q)}(y)}{W^{(q) \prime}\left(c_{2}^{*}\right)} \\
& \geq x-c_{2}^{*}+c_{2}^{*}-y-\beta,
\end{aligned}
$$

where the inequality follows from (10).

Since in the verification lemma below the Meyer-Itô formula is applied we require some smoothness on the value function of a $\left(c_{1} ; c_{2}\right)$ policy which in turn requires some smoothness on the scale function. We will call the scale function $W^{(q)}$ sufficiently smooth if $W^{(q)} \in C^{1}(0, \infty)$ when $X$ is of bounded variation. A necessary and sufficient condition for this is that the Lévy measure has no atoms (see [18, Theorem 2.9]). When $X$ is of unbounded variation we call the scale function sufficiently smooth if $W^{(q)} \in C^{1}(0, \infty)$ and $W^{(q) \prime}$ is absolutely continuous on $(0, \infty)$ with a density which is bounded on sets of the form $[1 / n, n], n \geq 1$. In [18, Theorem 2.6] it is proved that these latter conditions are fulfilled if the Gaussian coefficient $\sigma$ is strictly positive (the scale function is then even twice continuously differentiable) or if the Lévy measure has a log-convex density. Note that the term sufficiently smooth is used here in a slightly weaker sense than in [20].

We let $\Gamma$ be the operator defined by

$$
\Gamma f(x)=\gamma f^{\prime}(x)+\frac{\sigma^{2}}{2} f^{\prime \prime}(x)+\int_{0+}^{\infty}\left[f(x-y)-f(x)+f^{\prime}(x) y \mathbf{1}_{\{0<y<1\}}\right] \nu(\mathrm{d} y) .
$$

Here $x \in \mathbb{R}$ and $f$ is a function on $\mathbb{R}$ such that $\Gamma f(x)$ is well defined.

Lemma 6 (Verification lemma). Suppose the scale function $W^{(q)}$ is sufficiently smooth, $\left(c_{1}^{*}, c_{2}^{*}\right) \in C^{*}$ and

$$
(\Gamma-q) v_{c_{1}^{*}, c_{2}^{*}}(x) \leq 0
$$

for almost every $x>0$. Then $v_{c_{1}^{*}, c_{2}^{*}}=v_{*}(x)$ for all $x \geq 0$ and hence $\pi_{c_{1}^{*}, c_{2}^{*}}$ is an optimal strategy for the impulse control problem.

Proof. The proof is similar to the proofs of [18, Theorem 3.1] and [21, Lemma $5]$ and for the sake of brevity we only list the main differences.

1. We don't have that $v_{c_{1}^{*}, c_{2}^{*}}^{\prime}(x) \geq 1$ for all $x>0$ which is used in the proofs appearing in the two above mentioned references. Instead the inequality (9) should be employed. 
2. Since $v_{c_{1}^{*}, c_{2}^{*}}$ is not twice continuously differentiable, we cannot use anymore the standard Itô's formula when $X$ is of unbounded variation as in [21], but we have to appeal to the (extand second derivative) Meyer-Itô formula (cf. [23, Theorem IV.71]) as has been done in [18].

3. In $[18,21]$ inequality (11) has to hold for all $x>0$, whereas here we relaxed this in the sense that (11) needs to hold for almost every $x>0$. When the Gaussian coefficient $\sigma$ equals zero, these two conditions are equivalent thanks to the continuity of $(\Gamma-q) v_{c_{1}^{*}, c_{2}^{*}}(\mathrm{cf}$. [18, Lemma 4.1]). However, when $\sigma>0$, the left-derivative of $v_{c_{1}^{*}, c_{2}^{*}}^{\prime}$ at $c_{2}^{*}$ does not necessarily equal zero and therefore $v_{c_{1}^{*}, c_{2}^{*}}^{\prime \prime}\left(c_{2}^{*}\right)$ and thus $(\Gamma-q) v_{c_{1}^{*}, c_{2}^{*}}\left(c_{2}^{*}\right)$ are not well defined. We claim that when $\sigma>0$ the result still holds under the assumption that (11) only holds for almost every $x>0$.

Indeed, on inspection of [21, Lemma 5], we see that it is enough to show that for any $t>0$

$$
\int_{0}^{t} \mathrm{e}^{-q s}(\Gamma-q) v_{c_{1}^{*}, c_{2}^{*}}\left(\widetilde{U}_{s}^{c_{1}^{*}, c_{2}^{*}}\right) \mathrm{d} s \leq 0
$$

almost surely, where $\widetilde{U}^{c_{1}^{*}, c_{2}^{*}}$ is the right-continuous modification of $U^{c_{1}^{*}, c_{2}^{*}}$. Now let $A=\left\{x \in(0, \infty):(\Gamma-q) v_{c_{1}^{*}, c_{2}^{*}}(x)>0\right\}$ and $B=\{s \in[0, t]$ : $\left.\widetilde{U}_{s}^{c_{1}^{*}, c_{2}^{*}} \in A\right\}$. Since $\widetilde{U}^{c_{1}^{*}, c_{2}^{*}}$ is a semi-martingale we can use the occupation formula for the semi-martingale local time (see e.g. [23, Corollary 1, p.219]), to deduce a.s.

$$
\int_{0}^{t} \mathbf{1}_{\{s \in B\}} \sigma^{2} \mathrm{~d} s=\int_{0}^{t} \mathbf{1}_{\{s \in B\}} \mathrm{d}\left[\widetilde{U}^{c_{1}^{*}, c_{2}^{*}}, \widetilde{U}^{c_{1}^{*}, c_{2}^{*}}\right]_{s}^{c}=\int_{-\infty}^{\infty} \mathcal{L}_{t}^{a} \mathbf{1}_{\{a \in A\}} \mathrm{d} a
$$

with $\mathcal{L}^{a}$ being the semi-martingale local time at $a$ of $\widetilde{U} c_{1}^{*}, c_{2}^{*}$. Since by assumption $\sigma>0$ and $\operatorname{Leb}(A)=0(\operatorname{Leb}(\cdot)$ being the Lebesgue measure), it follows that $\operatorname{Leb}(B)=0$ and hence $(12)$ holds.

We now present the main result of this section.

Theorem 7. Suppose that $W^{(q)}$ is sufficiently smooth and that there exists $\left(c_{1}^{*} ; c_{2}^{*}\right) \in C^{*}$ such that

$$
W^{(q) \prime}(a) \leq W^{(q) \prime}(b) \quad \text { for all } c_{2}^{*} \leq a \leq b .
$$

Then the strategy $\pi_{c_{1}^{*}, c_{2}^{*}}$ is an optimal strategy for the impulse control problem.

Proof. From the verification lemma we see that it is enough to show that (11) holds for almost every $x>0$. First recall that by Corollary 4 we have $v_{c_{1}^{*}, c_{2}^{*}}=v_{c_{2}^{*}}$. Hence we can use e.g. [18, Lemma 4.2] to conclude that (11) holds for $x \in\left(0, c_{2}^{*}\right)$. For $x>c_{2}^{*}$ the property that $(\Gamma-q) v_{c_{2}^{*}}(x) \leq 0$ follows by mimicking the proof of Theorem 2 in Loeffen [20]. Note that it is here that one uses condition (13). 


\section{Optimal strategy when density is log-convex}

Throughout this section it is assumed that the Lévy measure has a log-convex density. Let $a^{\prime}$ be as in Proposition 1. We know then that $W^{(q) \prime}$ is strictly decreasing on $\left(0, a^{\prime}\right)$, constant on $\left(a^{\prime}, a^{*}\right)$ and strictly increasing on $\left(a^{*}, \infty\right)$. Moreover the scale function is sufficiently smooth and $W^{(q) \prime \prime+}(x)$ and $W^{(q) \prime \prime-}(x)$ exist for all $x>0$. Here $W^{(q) \prime \prime+}$ and $W^{(q) \prime \prime-}$ stand for respectively the rightand left-derivative of $W^{(q) \prime}$.

It is then easy to see from Proposition 3 and (8) that for each $\left(c_{1}^{*}, c_{2}^{*}\right) \in C^{*}$ we have $c_{1}^{*} \leq a^{\prime}$ and $c_{2}^{*}>a^{*}$ and hence by Theorem 7 the strategy $\pi_{c_{1}^{*}, c_{2}^{*}}$ is optimal. Indeed, when $c_{1}^{*}>0$ then $W^{(q) \prime}\left(c_{1}^{*}\right)=W^{(q) \prime}\left(c_{2}^{*}\right)$ and thus since $W^{(q) \prime}\left(c_{2}^{*}\right)>W^{(q)^{\prime}}\left(a^{*}\right)$ we must have $c_{1}^{*}<a^{\prime}$ and $c_{2}^{*}>a^{*}$. When $c_{1}^{*}=0$, then by $(7)$ and (8) it follows that $c_{2}^{*}$ cannot be smaller or equal to $a^{*}$.

Further it is straightforward to show that $C^{*}$ consists of only one element and hence there is a unique $\left(c_{1}, c_{2}\right)$ policy which is optimal for the control problem. Indeed, suppose that $\left(c_{1}, c_{2}\right)$ and $\left(c_{1}^{\prime}, c_{2}^{\prime}\right)$ are both in $C^{*}$. By (7) we then have $W^{(q) \prime}\left(c_{2}\right)=W^{(q) \prime}\left(c_{2}^{\prime}\right)$ and since $c_{2}, c_{2}^{\prime}>a^{*}$ and $W^{(q) \prime}$ is increasing on $\left(a^{*}, \infty\right)$, this implies that $c_{2}=c_{2}^{\prime}$. Similar arguments show that $c_{1}$ and $c_{1}^{\prime}$ can only be different if one of them is zero and the other strictly positive. Suppose without loss of generality that $c_{1}^{\prime}=0$ and $c_{1}>0$. Then by Proposition $3, W^{(q) \prime}\left(c_{1}\right)=W^{(q)^{\prime}}\left(c_{2}\right)=\left(W^{(q)}\left(c_{2}\right)-W^{(q)}(0)\right) /\left(c_{2}-\beta\right)$ and hence by using Proposition 3 again, the mean value theorem and $W^{(q) \prime}$ being strictly decreasing on $\left(0, c_{1}\right)$, we get the following contradiction

$$
\begin{aligned}
W^{(q) \prime}\left(c_{1}\right) & =\frac{\left\{W^{(q) \prime}\left(c_{1}\right)\left(c_{2}-\beta\right)+W^{(q)}(0)\right\}-W^{(q)}\left(c_{1}\right)}{c_{2}-c_{1}-\beta} \\
& =\frac{W^{(q) \prime}\left(c_{1}\right)\left(c_{2}-\beta\right)-W^{(q) \prime}(\xi) c_{1}}{c_{2}-c_{1}-\beta} \\
& <W^{(q) \prime}\left(c_{1}\right) .
\end{aligned}
$$

Here $\xi \in\left(0, c_{1}\right)$ is the number such that $W^{(q) \prime}(\xi) c_{1}=W^{(q)}\left(c_{1}\right)-W^{(q)}(0)$. It follows that $c_{1}$ has to be equal to $c_{1}^{\prime}$.

We now denote by $\left(c_{1}^{*}, c_{2}^{*}\right)$ the unique element of $C^{*}$ and give some conditions which specify whether $c_{1}^{*}=0$ or $c_{1}^{*}>0$. We first introduce some new functions and parameters. These will later also play a role in finding numerically the optimal parameters $c_{1}^{*}$ and $c_{2}^{*}$. The idea hereby is that due to Proposition 3 we can solve this 2 -dimensional minimization problem by solving two auxiliary 1-dimensional minimization problems.

Let $\varsigma_{2}:\left(0, a^{\prime}\right) \rightarrow\left(a^{*}, \infty\right)$ be the function implicitly defined by $W^{(q) \prime}(x)=$ $W^{(q)^{\prime}}\left(\varsigma_{2}(x)\right)$. Then $\varsigma_{2}$ is a strictly decreasing function. This together with the fact that $W^{(q)^{\prime}}$ is left- and right-differentiable and strictly increasing on $\left(a^{*}, \infty\right)$ 
implies that

$$
\begin{aligned}
\lim _{x \downarrow a} \frac{\varsigma_{2}(x)-\varsigma_{2}(a)}{x-a} & =\lim _{x \downarrow a} \frac{\varsigma_{2}(x)-\varsigma_{2}(a)}{W^{(q) \prime}\left(\varsigma_{2}(x)\right)-W^{(q) \prime}\left(\varsigma_{2}(a)\right)} \frac{W^{(q) \prime}(x)-W^{(q) \prime}(a)}{x-a} \\
& =\lim _{y \uparrow \varsigma_{2}(a)} \frac{y-\varsigma_{2}(a)}{W^{(q) \prime}(y)-W^{(q) \prime}\left(\varsigma_{2}(a)\right)} \lim _{x \downarrow a} \frac{W^{(q) \prime}(x)-W^{(q) \prime}(a)}{x-a} \\
& =\frac{W^{(q) \prime \prime}(a)}{W^{(q) \prime \prime}-\left(\varsigma_{2}(a)\right)}
\end{aligned}
$$

for $a \in\left(0, a^{\prime}\right)$ and thus $\varsigma_{2}$ is right-differentiable. (A similar calculation shows that $\varsigma_{2}$ is left-differentiable.) Note that from the proof of Proposition 1 it follows that $W^{(q) \prime \prime+}(x)<0$ for all $x<a^{*}$ and similarly we can deduce that $W^{(q) \prime \prime-}(x)>$ 0 for all $x>a^{*}$. Hence $\varsigma_{2}^{\prime+}(x)<0$ for all $x \in\left(0, a^{\prime}\right)$.

Let

$$
c_{1 \max }=\inf \left\{c_{1} \in\left(0, a^{\prime}\right): \varsigma_{2}\left(c_{1}\right)-c_{1} \leq \beta\right\},
$$

where we put $c_{1 \max }=0$ when $\lim _{x \downarrow 0} \varsigma_{2}(x) \leq \beta$ and then define the function $g_{1}:\left(0, c_{1 \max }\right) \rightarrow(0, \infty)$ by

$$
g_{1}\left(c_{1}\right)=g\left(c_{1}, \varsigma_{2}\left(c_{1}\right)\right)=\frac{W^{(q)}\left(\varsigma_{2}\left(c_{1}\right)\right)-W^{(q)}\left(c_{1}\right)}{\varsigma_{2}\left(c_{1}\right)-c_{1}-\beta} .
$$

Further define the function $g_{0}:(\beta, \infty) \rightarrow(0, \infty)$ by

$$
g_{0}\left(c_{2}\right)=g\left(0, c_{2}\right)=\frac{W^{(q)}\left(c_{2}\right)-W^{(q)}(0)}{c_{2}-\beta} .
$$

From the construction of the functions $g_{1}$ and $g_{0}$ and the existence of a unique minimizer for $g$, it is easy to see that if $c_{1}^{*}>0$, then $c_{1}^{*}$ is the unique minimizer of $g_{1}$ and that if $c_{1}^{*}=0$, then $c_{2}^{*}$ is the unique minimizer of $g_{0}$. For $g_{1}$ and $g_{0}$ we have the following differential equations

$$
\begin{aligned}
g_{1}^{\prime+}\left(c_{1}\right) & =\frac{\varsigma_{2}^{\prime+}\left(c_{1}\right)-1}{\varsigma_{2}\left(c_{1}\right)-c_{1}-\beta}\left(W^{(q) \prime}\left(c_{1}\right)-g_{1}\left(c_{1}\right)\right), \\
g_{0}^{\prime}\left(c_{2}\right) & =\frac{1}{c_{2}-\beta}\left(W^{(q) \prime}\left(c_{2}\right)-g_{0}\left(c_{2}\right)\right)
\end{aligned}
$$

and hence we get since $\varsigma_{2}^{\prime+}\left(c_{1}\right)<0$,

$$
\begin{aligned}
g_{1}^{\prime+}\left(c_{1}\right)<0(>0,=0) & \text { iff } \quad g_{1}\left(c_{1}\right)<W^{(q) \prime}\left(c_{1}\right)\left(>W^{(q) \prime}\left(c_{1}\right)=W^{(q) \prime}\left(c_{1}\right)\right), \\
g_{0}^{\prime}\left(c_{2}\right)<0(>0,=0) & \text { iff } \quad g_{0}\left(c_{2}\right)>W^{(q) \prime}\left(c_{2}\right)\left(<W^{(q) \prime}\left(c_{2}\right)=W^{(q) \prime}\left(c_{2}\right)\right) .
\end{aligned}
$$

We now show that $g_{0}$ has a unique minimizer. Note that $\lim _{x \downarrow \beta} g_{0}(x)=$ $\infty>W^{(q) \prime}(\beta)$ and that further for $x$ large enough, $g_{0}(x) \leq \frac{W^{(q)}(x)}{(1-\epsilon) x}$, for any $\epsilon \epsilon$ $(0,1)$ and by $(5) W^{(q) \prime}(x) \geq \Phi(q) W^{(q)}(x)$, which implies that $g_{0}(x)<W^{(q) \prime}(x)$ for $x$ large enough. This combined with (14), the behaviour of $W^{(q) \prime}$ and (8) 
implies that that there exists a unique point $\hat{c}_{2} \in\left(\beta \vee a^{*}, \infty\right)$ such that $g_{0}$ is strictly decreasing on $\left(\beta, \hat{c}_{2}\right)$ and strictly increasing on $\left(\hat{c}_{2}, \infty\right)$. Further, we have $g_{0}\left(\hat{c}_{2}\right)=W^{(q)^{\prime}}\left(\hat{c}_{2}\right)$. Hence if $c_{1}^{*}=0$, then $c_{2}^{*}=\hat{c}_{2}$.

Note that when $W^{(q) \prime}(0)<\infty$ and $a^{\prime}>0$, then $\varsigma_{2}(0):=\lim _{x \downarrow 0} \varsigma_{2}(x)<\infty$ and therefore the following parameter $\beta_{\max }$ is well defined,

$$
\beta_{\max }= \begin{cases}\infty & \text { if } W^{(q)^{\prime}}(0)=\infty \\ \varsigma_{2}(0)-\frac{W^{(q)}\left(\varsigma_{2}(0)\right)-W^{(q)}(0)}{W^{(q) \prime}(0)} & \text { if } W^{(q)^{\prime}}(0)<\infty \text { and } a^{\prime}>0 .\end{cases}
$$

Consider now the following three cases: (i) $a^{\prime}>0$ and $\beta<\beta_{\max }$, (ii) $a^{\prime}>0$ and $\beta \geq \beta_{\max }, \beta_{\max }<\infty$ and (iii) $a^{\prime}=0$.

Suppose we are in case (i). We show that then $g_{1}$ also has a unique minimizer. When $\beta_{\max }=\infty$, we have $W^{(q)^{\prime}}(0)=\infty$ and when $\beta_{\max }<\infty$, then

$$
g_{1}(0)=\frac{W^{(q)}\left(\varsigma_{2}(0)\right)-W^{(q)}(0)}{\varsigma_{2}(0)-\beta}<\frac{W^{(q)}\left(\varsigma_{2}(0)\right)-W^{(q)}(0)}{\varsigma_{2}(0)-\beta_{\max }}=W^{(q) \prime}(0) .
$$

This together with $\lim _{x \uparrow c_{1 \max }} g_{1}(x)=\infty>W^{(q) \prime}\left(c_{1 \max }\right)$, (14) and the fact that $W^{(q) \prime}$ is strictly decreasing on $\left(0, a^{\prime}\right)$ implies that there exists a unique point $\hat{c}_{1} \in\left(0, c_{1 \max }\right)$ such that $g_{1}$ is strictly decreasing on $\left(0, \hat{c}_{1}\right)$ and strictly increasing on $\left(\hat{c}_{1}, c_{1 \max }\right)$. Also $g_{1}\left(\hat{c}_{1}\right)=W^{(q) \prime}\left(\hat{c}_{1}\right)$.

From earlier considerations we now see that in case $(\mathrm{i}),\left(c_{1}^{*} ; c_{2}^{*}\right)$ is either equal to $\left(0 ; \hat{c}_{2}\right)$ or $\left(\hat{c}_{1} ; \varsigma_{2}\left(\hat{c}_{1}\right)\right)$. We will show that $\left(c_{1}^{*} ; c_{2}^{*}\right)$ is equal to the latter. First note that by (14) and $\hat{c}_{1}$ being strictly positive, we have $g_{1}\left(\hat{c}_{1}\right)=W^{(q)^{\prime}}\left(\hat{c}_{1}\right)<$ $W^{(q) \prime}(0)$. This implies that if $g_{0}\left(\hat{c}_{2}\right) \geq W^{(q) \prime}(0)$, then $g_{1}\left(\hat{c}_{1}\right)<g_{0}\left(\hat{c}_{2}\right)$ and so $g$ is minimized in $\left(\hat{c}_{1} ; \varsigma_{2}\left(\hat{c}_{1}\right)\right)$. Assume now that $g_{0}\left(\hat{c}_{2}\right)<W^{(q) \prime}(0)$. Then we have

$$
\lim _{x \downarrow 0} \frac{\partial}{\partial x} g\left(x, \hat{c}_{2}\right)=\frac{1}{\hat{c}_{2}-\beta}\left(g_{0}\left(\hat{c}_{2}\right)-W^{(q) \prime}(0)\right)<0
$$

and hence $g$ is not minimized in $\left(0 ; \hat{c}_{2}\right)$. It follows that $\left(c_{1}^{*} ; c_{2}^{*}\right)=\left(\hat{c}_{1} ; \varsigma_{2}\left(\hat{c}_{1}\right)\right)$.

Now assume that we are in case (ii). Then $\lim _{x \downarrow 0} g_{1}(x) \geq W^{(q) \prime}(0)$ and hence by (14) and the fact that $W^{(q) \prime}$ is strictly decreasing on $\left(0, a^{\prime}\right)$, we have that $g_{1}$ is strictly increasing on $\left(0, c_{1 \max }\right)$. Hence $\left(c_{1}^{*} ; c_{2}^{*}\right)=\left(0 ; \hat{c}_{2}\right)$.

Finally, suppose that we are in case (iii). Then $W^{(q) \prime}$ is an increasing function on $(0, \infty)$ and hence we conclude $\left(c_{1}^{*} ; c_{2}^{*}\right)=\left(0 ; \hat{c}_{2}\right)$.

We put the conclusions of this section in the following theorem.

Theorem 8. If the Lévy measure has a log-convex density, then there is a unique $\left(c_{1} ; c_{2}\right)$ policy which is optimal for the impulse control problem. Further, $c_{1}^{*}=0$ if and only if $\beta \geq \beta_{\max }$ or $a^{\prime}=0$, where $c_{1}^{*}$ is the unique optimal value of $c_{1}$.

\section{$5 \quad$ Examples}

In order to obtain the (candidate) optimal $\left(c_{1} ; c_{2}\right)$ policy one has to find the element(s) in $C^{*}$. In order to do this one first needs to evaluate the scale function 
which often has to be done by inverting the Laplace transform via numerical methods. But, even when there is a (simple) explicit expression for the scale function, it is not possible to give an explicit formula for the optimal parameters $c_{1}^{*}$ and $c_{2}^{*}$ (if they exist); see e.g. Theorem B in Jeanblanc and Shiryaev [14] for the case when $X$ is a Brownian motion plus drift. Hence one has to resort to numerical methods to find the minimizer(s) of $g$. One possibility is to minimize the function $g$ over $c_{1}$ and $c_{2}$ via a numerical program, but it might be that one ends up with a local instead of a global minimum. However, from the previous section we know that when the Lévy measure has a log-convex density, we can find the optimal parameters by minimizing either $g_{0}$ or $g_{1}$, both being functions of just one variable and with only one local minimum. In case the Lévy measure does not have a log-convex density, the element(s) of $C^{*}$ might still be found by applying some of the methods described in Section 4 locally. We give an example of both cases. The figures and calculations in these examples are all made with the help of Matlab.

Example 1 The first example concerns the case when $X$ is a spectrally negative stable process with index $\alpha \in(1,2)$. Its Laplace exponent is given by $\psi(\theta)=\theta^{\alpha}$. An explicit expression for its scale function was found by Bertoin [6] and is given by $W^{(q)}(x)=\alpha x^{\alpha-1} E_{\alpha}^{\prime}\left(q x^{\alpha}\right)$ for $q, x \geq 0$, where $E_{\alpha}^{\prime}$ is the derivative of the Mittag-Leffler function of index $\alpha$ given by $E_{\alpha}(x)=\sum_{n=0}^{\infty} \frac{x^{n}}{G(1+\alpha n)}$ with $G(\cdot)$ being the gamma function. Since the Lévy measure of this process, given by $\nu(\mathrm{d} x)=\frac{\alpha(\alpha-1)}{G(2-\alpha)} x^{-1-\alpha} \mathrm{d} x$, has a completely monotone density and $W^{(q) \prime}(0)=\infty=\beta_{\max }$, we know by Theorem 8 that the set $C^{*}$ consists of exactly one point, denoted by $\left(c_{1}^{*} ; c_{2}^{*}\right)$, that $c_{1}^{*}>0$ and that the strategy $\pi_{c_{1}^{*}, c_{2}^{*}}$ is optimal for the impulse control problem. Further we know from Section 4 that $c_{1}^{*}$ is the unique minimum of the function $g_{1}$ on $\left(0, c_{1 \text { max }}\right)$ and the only intersection point of $W^{(q) \prime}$ and $g_{1}$; moreover, $c_{2}^{*}$ is given by the unique point in $\left(a^{*}, \infty\right)$ such that $W^{(q) \prime}\left(c_{2}^{*}\right)=W^{(q) \prime}\left(c_{1}^{*}\right)$. In our example the parameters are chosen as follows: $\alpha=1.5, q=0.1$ and $\beta=1$. In Figure 1 the graphs of $W^{(q) \prime}$ and $g_{1}$ are plotted and the optimal levels are found to be equal to $\left(c_{1}^{*} ; c_{2}^{*}\right)=(0.41 ; 4.85)$, whereas the parameter $c_{1 \max }=1.33$.

Example 2 For the second example we consider, we let $X$ be a CramérLundberg risk process as in (1) with Lévy measure given by $\nu(\mathrm{d} x)=\lambda \alpha^{2} x \mathrm{e}^{-\alpha x}$. This means that the claims are $\operatorname{Erlang}(2, \alpha)$ distributed. The scale function for $X$, which can be derived by the method of partial fraction, is given by

$$
W^{(q)}(x)=\sum_{i=1}^{3} D_{i} \mathrm{e}^{\theta_{i} x}, \quad x \geq 0,
$$

where $\left\{\theta_{i}: i=1,2,3\right\}$ are the (distinct) roots of

$$
\psi(\theta)-q=c \theta-\lambda+\frac{\lambda \alpha^{2}}{(\alpha+\theta)^{2}}-q,
$$




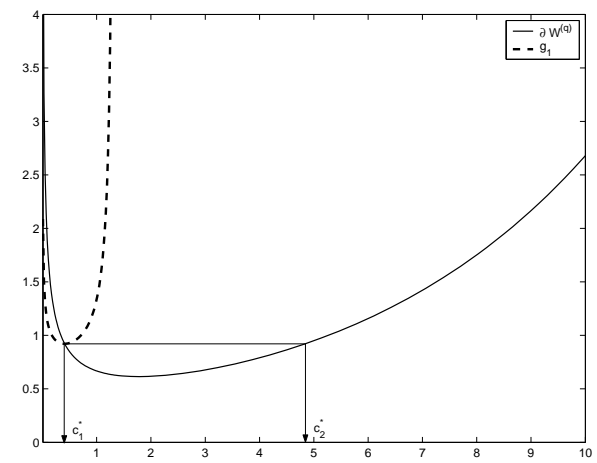

Figure 1: Stable with index 1.5

with $\theta_{1}>0$ and $\theta_{2}, \theta_{3}<0$ and where $\left\{D_{i}: i=1,2,3\right\}$ are given by $D_{i}=$ $1 / \psi^{\prime}\left(\theta_{i}\right)$. We now choose the parameters as follows: $c=21.4, \lambda=10, \alpha=1$, $q=0.1$ and for $\beta$ we consider two cases, the case when $\beta=0.015$ and $\beta=0.2$. This example corresponds to the one in Azcue and Muler [5] for which they showed that the optimal strategy for the de Finetti problem is not a barrier strategy. Note that the Lévy measure does not have a log-convex density and therefore Theorem 8 does not apply.

The derivative of this scale function is plotted in Figure 2. We see from Figure 2 that the absolute minimum of $W^{(q) \prime}$ is attained at $x=0$, but that this function further also has a local maximum and a second local minimum. Denote by $a_{1}$ resp. $a_{2}$ the point on the $x$-axis at which $W^{(q) \prime}$ has this local maximum resp. local minimum. Further denote by $\varsigma\left(a_{1}\right) \in\left(a_{2}, \infty\right)$ the point such that $W^{(q) \prime}\left(\varsigma\left(a_{1}\right)\right)=W^{(q)^{\prime}}\left(a_{1}\right)$ and by $\varsigma\left(a_{2}\right) \in\left(0, a_{1}\right)$ the point such that $W^{(q) \prime}\left(\varsigma\left(a_{2}\right)\right)=W^{(q)^{\prime}}\left(a_{2}\right)$. Note that one can see from the figure that these points exist.

We now want to find for a given $\beta$ the elements of $C^{*}$; we can then use Theorem 7 to find out if a certain $\left(c_{1} ; c_{2}\right)$ policy is optimal. Let $\left(c_{1}^{*} ; c_{2}^{*}\right) \in$ $C^{*}$. Since by Proposition 3 we must have $c_{1}^{*}=0$ or $W^{(q) \prime}\left(c_{1}^{*}\right)=W^{(q) \prime}\left(c_{2}^{*}\right)$, it follows that there are three possible cases: (i) $c_{1}^{*}=0$, (ii) $c_{2}^{*} \in\left(a_{2}, \varsigma\left(a_{1}\right)\right)$, $c_{1}^{*} \in\left(\varsigma\left(a_{2}\right), a_{2}\right)$ and (iii) $c_{2}^{*} \in\left(a_{1}, a_{2}\right), c_{1}^{*} \in\left(\varsigma\left(a_{2}\right), a_{1}\right)$. Though we will now show that (iii) cannot happen. Let $\tilde{\varsigma}_{2}:\left(\varsigma\left(a_{2}\right), a_{1}\right) \rightarrow\left(a_{1}, a_{2}\right)$ be the function implicitly defined by $W^{(q)^{\prime}}\left(\tilde{\varsigma}_{2}(x)\right)=W^{(q) \prime}(x)$ and let $\tilde{g}_{1}(x)=g\left(x, \tilde{\varsigma}_{2}(x)\right)=$ $\frac{W^{(q)}\left(\tilde{\varsigma}_{2}(x)\right)-W^{(q)}(x)}{\tilde{\varsigma}_{2}(x)-x-\beta}$, where we take the domain of $\tilde{g}_{1}$ to be all $x \in\left(\varsigma\left(a_{2}\right), a_{1}\right)$ big enough such that the denominator of $\tilde{g}_{1}(x)$ is strictly positive. By the mean value theorem and the fact that $W^{(q) \prime}$ is strictly increasing on $\left(\varsigma\left(a_{2}\right), a_{1}\right)$ and strictly decreasing on $\left(a_{1}, a_{2}\right)$, we get for all $x$ in the domain of $\tilde{g}_{1}$

$$
\tilde{g}_{1}(x) \geq \min _{\xi \in\left[x, \tilde{\varsigma}_{2}(x)\right]} W^{(q)^{\prime}}(\xi) \frac{\tilde{\varsigma}_{2}(x)-x}{\tilde{\varsigma}_{2}(x)-x-\beta}>W^{(q)^{\prime}}(x)
$$

and thus by Proposition 3, case (iii) is not possible. 
This leaves the remaining two cases (i) and (ii). To find out which value(s) $\left(c_{1}^{*} ; c_{2}^{*}\right)$ takes, we introduce the function $\varsigma_{2}:\left(\varsigma\left(a_{2}\right), a_{2}\right) \rightarrow\left(a_{2}, \varsigma\left(a_{1}\right)\right)$ implicitly defined by $W^{(q) \prime}\left(\varsigma_{2}(x)\right)=W^{(q) \prime}(x)$ and let

$$
D=\left\{c_{1} \in\left(\varsigma\left(a_{2}\right), a_{2}\right): \varsigma_{2}\left(c_{1}\right)-c_{1}>\beta\right\} .
$$

As in Section 4, we define the functions $g_{1}: D \rightarrow(0, \infty)$ and $g_{0}:(\beta, \infty) \rightarrow$ $(0, \infty)$ given by $g_{1}(x)=g\left(x, \varsigma_{2}(x)\right)$ and $g_{0}(x)=g(0, x)$. Note that the minimum of $g$ will be equal to either the minimum of $g_{0}$ or to the minimum of $g_{1}$, whichever one lies lower; in case (i) the minimum of $g_{0}$ will lie lower, in case (ii) it will be the other way around.

In Figure 2 the graphs of $g_{0}, g_{1}$ and $W^{(q) \prime}$ are plotted for both values of $\beta$. For $\beta=0.015$, we see that the minimum of $g_{0}$ lies lower than the minimum of $g_{1}$ and hence $c_{1}^{*}=0$; the other level is then found to be equal to $c_{2}^{*}=0.316$. Further we see that condition (13) of Theorem 7 is not satisfied. Hence we cannot conclude at this stage that an optimal strategy for the impulse control problem is formed by a $\left(c_{1} ; c_{2}\right)$ policy.
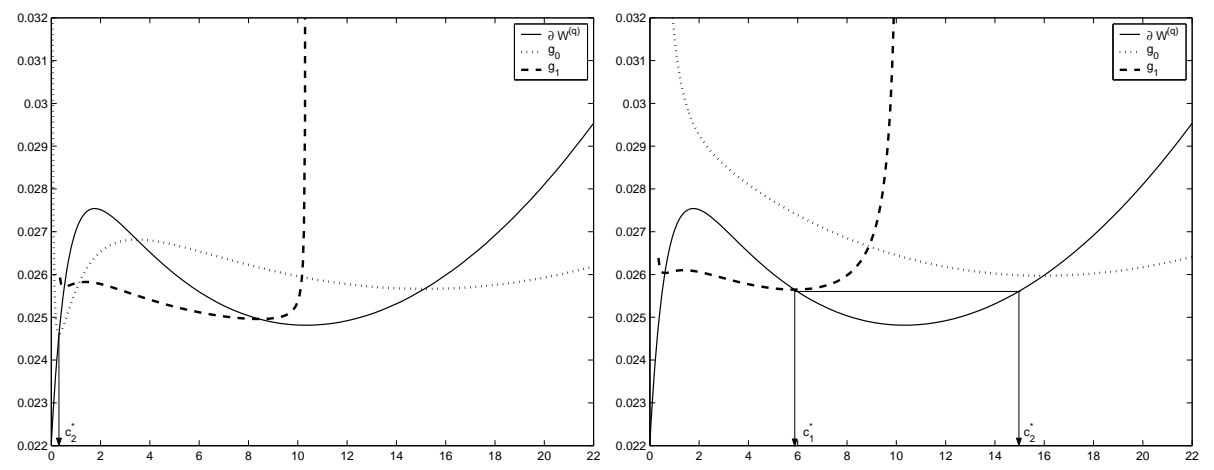

Figure 2: Cramér-Lundberg with $\operatorname{Erlang}(2,1)$ claims; left: $\beta=0.015$, right: $\beta=0.2$

But this does not mean that the strategy $\pi_{c_{1}^{*}, c_{2}^{*}}$ is not optimal, since Theorem 7 only gives sufficient, and not necessary, conditions for a particular $\left(c_{1} ; c_{2}\right)$ policy to be optimal. To see that actually no $\left(c_{1} ; c_{2}\right)$ policy is optimal for the impulse control problem, we first note that by the representation for the value function of a $\left(c_{1} ; c_{2}\right)$ policy given in Proposition 2 and the fact that $\left(c_{1}^{*} ; c_{2}^{*}\right)$ is the only minimizer of $g$, that for all $\left(c_{1} ; c_{2}\right) \neq\left(c_{1}^{*} ; c_{2}^{*}\right)$,

$$
v_{c_{1}^{*}, c_{2}^{*}}(x)>v_{c_{1}, c_{2}}(x) \text { for all } 0<x \leq c_{2}^{*} \wedge c_{2}
$$

and hence the only $\left(c_{1} ; c_{2}\right)$ policy which can be optimal is the one with the levels equal to $c_{1}^{*}$ and $c_{2}^{*}$. But if the parameters $c_{1}^{\prime}=8$ and $c_{2}^{\prime}=12$ are taken, one can calculate that for the starting value $x=6$,

$$
v_{c_{1}^{\prime}, c_{2}^{\prime}}(6)=8.235>7.883=v_{c_{1}^{*}, c_{2}^{*}}(6)
$$


and thus $\pi_{c_{1}^{*}, c_{2}^{*}}$ is not optimal and therefore no $\left(c_{1} ; c_{2}\right)$ policy is optimal for the impulse control problem in this case.

In the other case when $\beta=0.2$, we see that $\left(c_{1}^{*} ; c_{2}^{*}\right)$ satisfies (13) and hence we can conclude that $\pi_{c_{1}^{*}, c_{2}^{*}}$ is an optimal strategy for the impulse control problem.

\section{References}

[1] H. Albrecher, J.-F. Renaud, and X. Zhou, A Lévy insurance risk process with tax, Journal of Applied Probability 45 (2008), no. 2, 363-375.

[2] L.H.R. Alvarez and T.A. Rakkolainen, Optimal payout policy in presence of downside risk (2008), To appear in Mathematical Methods of Operations Research.

[3] J.A. Bather, A continuous time inventory model, Journal of Applied Probability 3 (1966), 538-549.

[4] F. Avram, Z. Palmowski, and M.R. Pistorius, On the optimal dividend problem for a spectrally negative Lévy process, Annals of Applied Probability 17 (2007), 156-180.

[5] P. Azcue and N. Muler, Optimal reinsurance and dividend distribution policies in the Cramér-Lundberg model, Mathematical Finance 15 (2005), 261-308.

[6] J. Bertoin, On the first exit time of a completely asymmetric Lévy process from a finite interval, Bulletin of the London Mathematical Society 28 (1995), 514-520.

[7] B. de Finetti, Su un'impostazion alternativa dell teoria collecttiva del rischio, Transactions of the XVth International Congress of Actuaries 2 (1957), 433-443.

[8] F. Dufresne and Gerber H.U., The probability of ruin for the inverse Gaussian and related processes, Insurance: Mathematics and Economics 12 (1993), 9-22.

[9] F. Dufresne, Gerber H.U., and E.S.W. Shiu, Risk theory with the gamma process, Astin Bulletin 21 (1991), 177-192.

[10] H. Furrer, Risk processes perturbed by a-stable Lévy motion, Scandinavian Actuarial Journal (1998), 59-74.

[11] H.U. Gerber, Entscheidungskriterien für den zusammengesetzten Poisson-Prozess, Mitteilungen der Vereinigung Schweizerischer Versicherungsmathematiker 69 (1969), 185227.

[12] F. Hubalek and A.E. Kyprianou, Old and new examples of scale functions for spectrally negative Lévy processes (2007). Preprint.

[13] M. Huzak, M. Perman, H. Šikić, and Z. Vondraček, Ruin probabilities and decompositions for general perturbed risk processes, The Annals of Applied Probability 14 (2004), 13781397.

[14] M. Jeanblanc-Picqué and A.N. Shiryaev, Optimization of the flow of dividends, Russian Math. Surveys 50 (1995), 257-277.

[15] A.E. Kyprianou, Introductory lectures on fluctuations of Lévy processes with applications, Springer, 2006.

[16] A.E. Kyprianou and Z. Palmowski, Distributional study of de Finetti's dividend problem for a general Lévy insurance risk process, Journal of Applied Probability 44 (2007), 349-365.

[17] A.E. Kyprianou and V. Rivero, Special, conjugate and complete scale functions for Spectrally negative Lévy processes, Electronic Journal of Probability 13 (2008), no. 57, 16721701.

[18] A.E. Kyprianou, V. Rivero, and R. Song, Smoothness and convexity of scale functions with applications to de Finetti's control problem, 2008. arXiv:0801.1951v2 [math.PR]. 
[19] A.E. Kyprianou and B.A. Surya, Principles of smooth and continuous fit in the determination of endogenous bankruptcy levels, Finance and Stochastics 11 (2007), no. 1, 131-152.

[20] R.L. Loeffen, On optimality of the barrier strategy in de Finetti's dividend problem for spectrally negative Lévy processes, Annals of Applied Probability 18 (2008), no. 5, 16691680.

[21] _ An optimal dividends problem with a terminal value for spectrally negative Lévy processes with a completely monotone jump density (2008), To appear in Journal of Applied Probability.

[22] J. Paulsen, Optimal dividend payments until ruin of diffusion processes when payments are subject to both fixed and proportional costs, Advances in Applied Probability 39 (2007), 669-689.

[23] P. Protter, Stochastic integration and differential equations, 2nd ed., version 2.1, Springer, 2005.

[24] J.F. Renaud and X. Zhou, Distribution of the present value of dividend payments in a Lévy risk model, Journal of Applied Probability 44 (2007), 420-427.

[25] A. Sulem, A solvable one-dimensional model of a diffusion inventory system, Mathematics of Operations Research 11 (1986), no. 1, 125-133.

[26] B.A. Surya, Evaluating scale functions of spectrally negative Lévy processes, Journal of Applied Probability 45 (2008), no. 1, 135-149. 